\title{
Patterned growth of pentacene
}

\author{
Soeren Steudel, ${ }^{\text {a),b) }}$ Dimitri Janssen, ${ }^{\text {c) }}$ Stijn Verlaak, ${ }^{\text {b) }}$ Jan Genoe, and Paul Heremans ${ }^{\text {b) }}$ \\ IMEC, Polymer and Molecular Electronics, Kapeldreef 75, B-3001 Leuven, Belgium
}

(Received 5 May 2004; accepted 13 October 2004)

\begin{abstract}
We propose a way of patterning organic small molecule thin films without requiring a hardmask and therefore more compatible with printing technologies. Active and passive areas for transistors are predefined by different surface chemistries. The subsequent growth takes place under conditions that cause the formation of a high mobility two-dimensional film in the active area and a disconnected three-dimensional film or no film in the passive area. This concept is founded on the basic theory of nucleation of organic small molecules on inert substrates and applied to the growth of patterned pentacene layers. (C) 2004 American Institute of Physics. [DOI: 10.1063/1.1832732]
\end{abstract}

Organic thin film transistors are promising for cheap, flexible, large area applications. There are two groups of organic semiconducting materials-polymers and small molecules. The advantage of polymers lays in the simple solution process-ability that allows the use of cheap printing technologies, whereas evaporated small molecules show superior mobility comparable to amorphous silicon. Depending on the application, both approaches are potentially interesting.

To prevent cross-talk between transistors in close proximity and to achieve a low off-current, it is necessary to pattern the organic semiconductor layer. Several ways to pattern small molecules are already published, e.g., evaporation of the small molecules through a shadow mask, ${ }^{1}$ deposition of a water-soluble resist on top of the organic semiconductor followed by lithographic patterning, ${ }^{2}$ as well as using an integrated shadow mask whereby a resist is lithographically patterned to achieve step edges around the intended active area. ${ }^{3}$ In the latter, the subsequently evaporated small molecule layer is discontinued by the step edges of the resist around the active area. Those ways of patterning small molecule layers all have in common that they require a maskeither a shadow mask or a lithographic mask - to be aligned. The use of a hard mask reduces the flexibility of the design. There are further drawbacks connected with the abovementioned ways of patterning. For instance, the patterning with water-soluble resist results in some degradation of the transistor characteristics.

This letter proposes a way of patterning films of small molecules that is compatible with printing techniques. In this technique, we predefine active and passive parts on the substrate area by giving them different surface treatments. In the subsequent vacuum growth, the small molecules form a high-mobility two-dimensional (2D) film on the active part and an ill-connected low-mobility three-dimensional (3D) film (or even no film at all) on the passive area.

A variety of papers which have different perspectives on surface modification as a way to template growth ${ }^{4,5}$ and to influence the morphology and electrical characteristics ${ }^{6-10}$ of thin films of small organic molecules have been published before. In our letter, results showing the possibility to pattern pentacene films by surface modification will be presented in

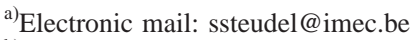

${ }^{b}$ Also with E. E. Dept. of K. U. Leuven.

${ }^{c)}$ Also with Chem. Dept. of K. U. Leuven.
}

the framework of an "atomistic" theory of growth. ${ }^{11}$

When molecules are deposited on a substrate, those molecules aggregate and form a nucleus. Small nuclei are unstable because of their surface energy and an energetic barrier against nucleation should be overcome before a nucleus has gained enough "mass" to become stable. While growing further, the nucleus will become less unstable and will finally be stable. At high substrate temperatures $T_{\text {sub }}$ and low fluxes $\Phi$ (i.e., low supersaturation $\Delta \mu$ ), many molecules are required to form a stable nucleus, and this nucleus will be several monolayers high (3D layer). ${ }^{12}$ In this case, the energy barrier against nucleation is given by ${ }^{11}$

$$
\Delta G_{3 \mathrm{D}}=\frac{2\left(\Psi_{c}-\Psi_{\text {mol-sub }}\right) g\left(\Psi_{i j k}\right)}{\Delta \mu^{2}} .
$$

Here, $\Psi_{i j k}$ is the surface energy per unit cell of surface $(i j k)$, which has been calculated elsewhere, ${ }^{11} \Psi_{c}$ is the interaction between 1 molecule and the neighboring (001) plane; ${ }^{11} \Psi_{\text {mol-sub }}$ is the interaction between that molecule and the substrate. The supersaturation is defined as the difference in chemical potential between a molecule in the vapor and a molecule in a bulk crystal. This supersaturation depends on the growth or deposition conditions. For the growth from vapor the supersaturation can be expressed as ${ }^{13}$

$$
\Delta \mu \approx R T_{\text {sub }} \operatorname{In}\left(\frac{\sqrt{2 \pi M R T_{\text {sub }}} \Phi}{P_{\infty}\left(T_{\text {sub }}\right)}\right),
$$

where $R$ is the universal gas constant, $M$ the weight of the pentacene molecule, and $P_{\infty}\left(T_{\text {sub }}\right)$ the equilibrium standard vapor pressure of pentacene at temperature $T_{\text {sub }}$. The vapor pressure will vary at parts of the substrate that are treated differently, due to thermal desorption. This effect is not taken into account in Eq. (2), and inevitably introduces some error in the determination of $\Delta \mu$. In any case, height cross sections will show that a change in nucleation mode rather than nucleation density is the dominant effect in patterned growth. In addition, Eq. (2) has been demonstrated to acceptably predict variation with $T_{\text {sub }}$ and flux. ${ }^{11}$

It is clear that the higher the supersaturation, i.e., the more energy the system loses during energy minimization by incorporating a molecule into the nucleus, the fewer molecules are required to form a stable nucleus. At sufficiently high supersaturation, a stable nucleus will only be one monolayer high, and the energy barrier against this twodimensional nucleation is now given by ${ }^{11}$ 


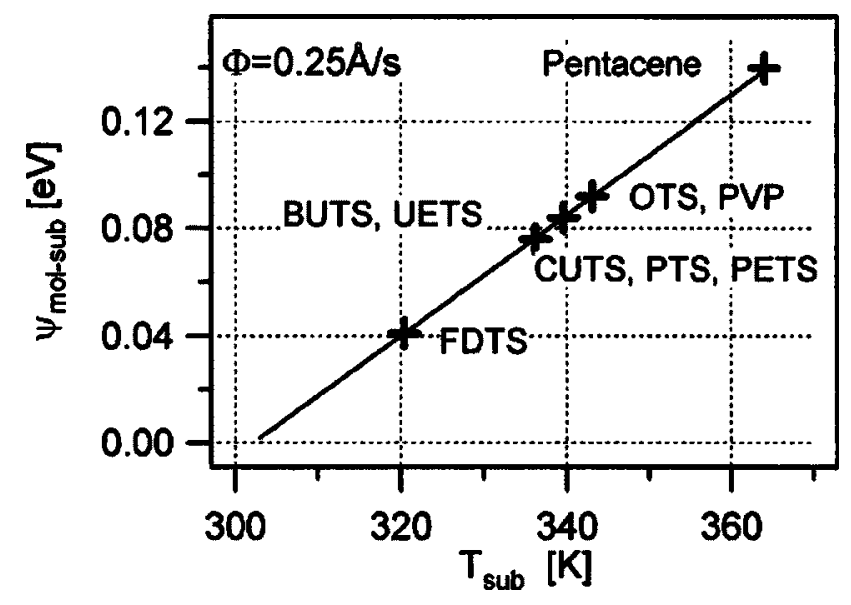

FIG. 1. Pentacene-substrate interaction for different surface treatments: PTS-Phenyltrichlorosilane; PETS—Phenylethyltrichlorosilane; UETS10-Undecenyltrichlorosilane; OTS-Octadecyltrichlorosilane; FDTS(Heptadecafluoro-1,1,2,2-tetrahydrodecyl)trichlorosilane; BUTS-11Bromoundecyltrichlorosilane, CUTS-11-Cyanoundecyltrichlorosilane.

$$
\Delta G_{2 \mathrm{D}}=\frac{g\left(\Psi_{i j k}\right)}{2 \Delta \mu-2\left(\Psi_{c}-\Psi_{\text {mol-sub }}\right)} .
$$

While for low supersaturations it will be more favorable to nucleate three-dimensional, there is a transition at a supersaturation $\Delta \mu_{\mathrm{tr}}$ at which $\Delta G_{3 \mathrm{D}}=\Delta G_{2 \mathrm{D}}$. Above $\Delta \mu_{\mathrm{tr}}, \Delta G_{3 \mathrm{D}}$ would still be smaller than $\Delta G_{2 \mathrm{D}}$, yet becomes physically meaningless since it corresponds to a nuclei less than one monolayer high. By changing the surface chemistry the strength of the small molecule-surface interaction $\Psi_{\text {mol-sub }}$ can be influenced and therefore the window for $2 \mathrm{D}$ and $3 \mathrm{D}$ growth. The strength of this interaction is specific for every small molecule-surface combination and is not necessarily related, e.g., to surface hydrophobicity as was suggested by Kosbar et al. ${ }^{10}$ To achieve a high mobility, the formation of a well-connected 2D layer is a pre-requirement.

To get an overview of the range over which the molecule-surface interaction can be varied we deposited $30 \mathrm{~nm}$ pentacene ( $\Phi \sim 0.25 \AA / \mathrm{s}, \mathrm{p} \sim 7 \mathrm{e}-7$ Torr $)$ on thermal grown $\mathrm{SiO}_{2}$. A quartz crystal monitor held at $297 \mathrm{~K}$ was used for measuring the flux. Applying a self-assembled monolayer (SAM) beforehand, the surface of the $\mathrm{SiO}_{2}$ was modified. This guarantees that the chemical properties of the surface are modified without changing the roughness of the surface.

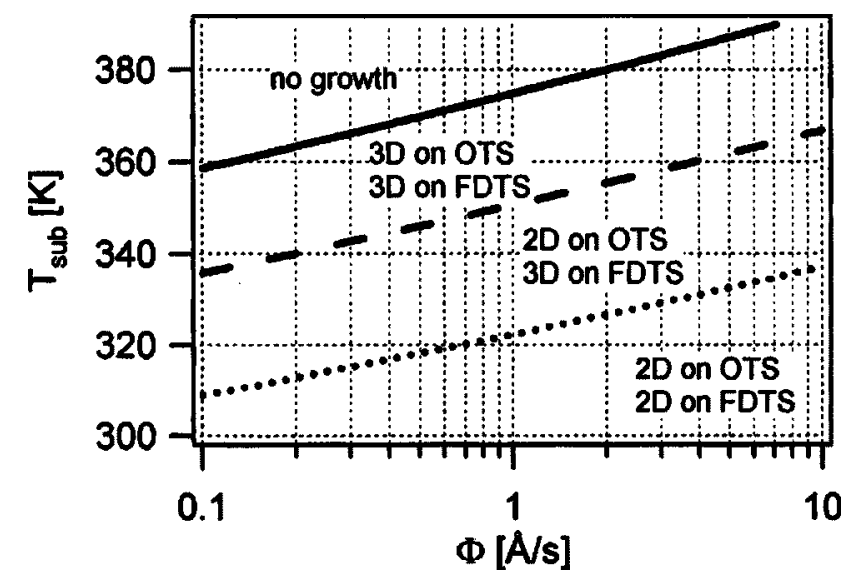

FIG. 2. Growth window for pentacene on OTS/FDTS.

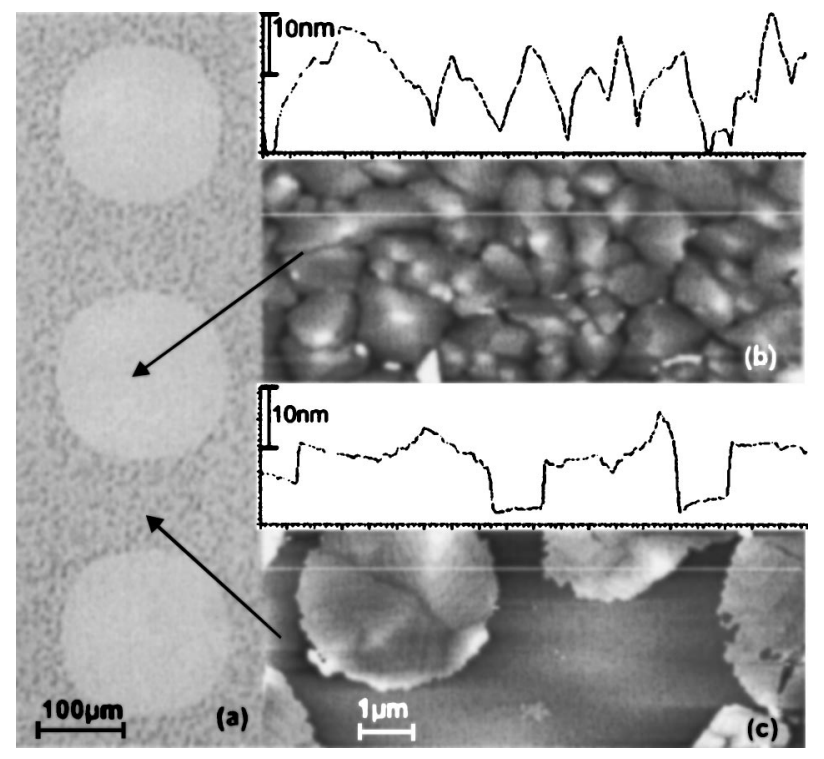

FIG. 3. Patterned growth of pentacene on FDTS/OTS - substrate temperature $=338 \mathrm{~K}$ : (a) optical image of patterned pentacene, (b) AFM image with height cross section of continued 2D film on OTS, (c) AFM image with height cross section of disconnected 3D film on FDTS.

First, the substrates have been cleaned for 5 min by UV-ozone. Next, the SAMs (PTS-Phenyltrichlorosilane, PETS-Phenylethyltrichlorosilane, UETS-10-Undecenyltrichlorosilane, OTS-Octadecyltrichlorosilane, FDTS(Heptadecafluoro-1,1,2,2 - tetrahydrodecyl)trichlorosilane, BUTS-11-Bromoundecyltrichlorosilane, CUTS-11Cyanoundecyltrichlorosilane) were deposited out of vapor phase in a low pressure furnace (10 $\mathrm{mbar}, 140^{\circ}$, and $45 \mathrm{~min}$ ). The high quality of the layer can be seen by water contact angle measurements which yield values of $\sim 110^{\circ}$. No polymerized residues of the silane can be found by atomic force microscope (AFM). Pentacene was subsequently deposited at different substrate temperatures. With help of AFM measurements the transition from $2 \mathrm{D}$ to $3 \mathrm{D}$ growth was determined and the substrate temperature at this transition point was calculated back to a molecule-surface interaction value $\Psi_{\text {mol-sub }}$ (Fig. 1). 2D growth was defined as growth of monolayer high terraces (1.5 nm for pentacene) from the substrate whereby $3 \mathrm{D}$ growth shows steep multiple layer high edges. ${ }^{11}$

The biggest differences in pentacene-substrate interactions can be found according to Fig. 1 between pentacene on OTS and pentacene on FDTS. Those two surface-treatments therefore offer the largest window of growth conditions for patterned growth. The available process window, assuming in the previous experiment extracted values for substrate to pentacene interactions, has been calculated in Fig. 2. The relation between 2D, 3D, and no growth for pentacene molecules on OTS and FDTS depending on the deposition conditions can be seen in this figure.

The next step of the experiment involved generating patterns with an OTS SAM, while covering the remainder of the substrate by a FDTS SAM. The patterning of the SAMs is done in the following way: FDTS was deposited on Si wafers with thermally grown $\mathrm{SiO}_{2}$. Next, a shadow mask was placed on the samples and the FDTS was etched away on the uncovered regions with UV-ozone $(5 \mathrm{~min}$ ). Subsequently, OTS was deposited. Because the OTS can attach itself only to $\mathrm{SiO}_{2}$ and not where FDTS is remaining, patterned regions with only FDTS or only OTS can be achieved. Subsequently, 


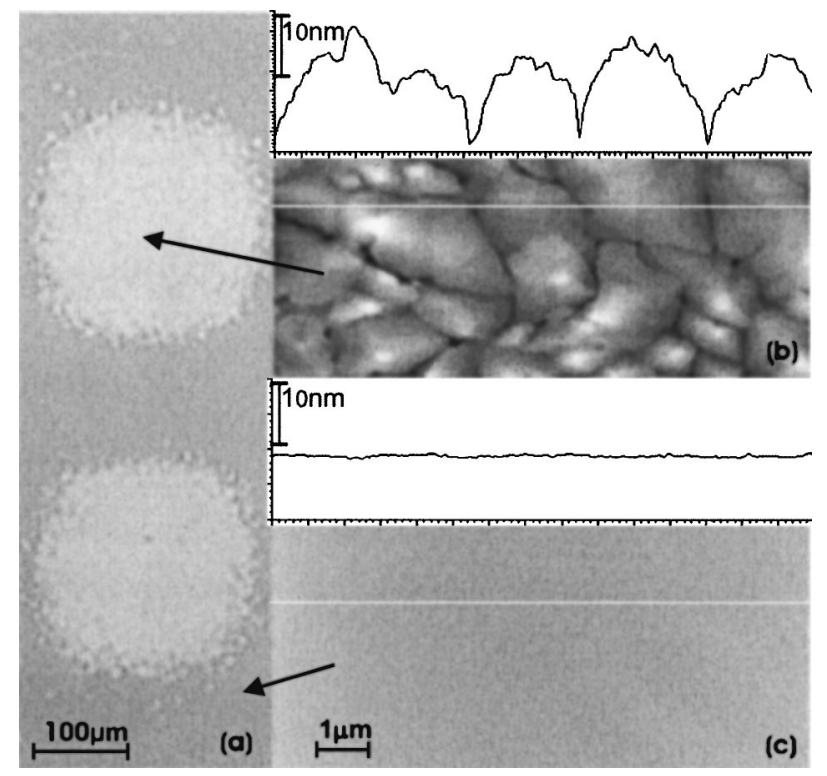

FIG. 4. Patterned growth of pentacene on FDTS/OTS - substrate temperature $=345 \mathrm{~K}$ : (a) optical image of patterned pentacene, (b) AFM image with height cross section of continued 2D film on OTS, (c) AFM image with height cross section without any nucleation on FDTS.

pentacene was deposited at substrate temperatures just below the upper limit for 2D nucleation on OTS at a flux of $0.25 \AA / s$. In a broad substrate-temperature range $\left(\Delta T_{\text {sub }}\right.$ $\sim 20 \mathrm{~K})$ the pentacene on OTS grew as a 2D layer with a mobility of $\mu \sim 0.6 \mathrm{~cm}^{2} / \mathrm{V}$ s while the pentacene on FDTS formed a disconnected 3D layer with a mobility of $\mu<0.06 \mathrm{~cm}^{2} / \mathrm{V}$ s (Fig. 3). To perform electrical measurements, Au source-drain contacts were deposited through a shadow mask and the mobility was extracted from the transfer characteristic of the transistor in saturation.

At even higher substrate temperatures $T_{\text {sub }} \sim 345 \mathrm{~K}$ a continuous 2D film was formed on the OTS and only few disconnected single grains could be seen on the FDTS areas (Fig. 4). However, the temperature window to obtain this effect on FDTS/OTS is very small $\left(\Delta T_{\text {sub }} \sim 2 \mathrm{~K}\right)$.
To gain a stable process window for patterned growth, further investigation into the interaction between surface and small molecule will be necessary. In particular, polymer films are interesting as growth surface because of the possibility to influence not only the small molecule-surface interaction but at the same time to use the polymers as gatedielectric.

In summary, a patterning technique-patterned growth-for small organic molecules based on an atomistic theory of nucleation was demonstrated and verified for pentacene. This technique allows the controlled nucleation of evaporated small molecules depending on the local surface chemistry and therefore opens the opportunity to combine printing techniques like micro-contact printing or ink-jet printing with evaporated small molecules.

The author would like to thank the European Union for financial support through the project NAIMO, IP 500355.

${ }^{1}$ P. F. Baude, D. A. Ender, M. A. Haase, T. W. Kelly, D. V. Muyres, and S. D. Theiss, Appl. Phys. Lett. 82, 22 (2003).

${ }^{2}$ M. G. Kane, J. Campi, M. S. Hammond, B. Greening, C. D. Sheraw, J. A. Nichols, D. J. Gundlach, J. R. Huang, C. C. Kuo, L. Jia, H. Klauk, and T. N. Jackson, IEEE Electron Device Lett. 21, 11 (2000).

${ }^{3}$ C. Py, D. Roth, I. Levesque, J. Stabledon, and A. Donat-Buillud, Synth. Met. 122, 225 (2001).

${ }^{4}$ H. Y. Choi, S. H. Kim, and J. Jang, Adv. Mater. (Weinheim, Ger.) 16, 732 (2004).

${ }^{5}$ M. Ando, M. Kawasaki, and S. Imazeki, Appl. Phys. Lett. 85, 6 (2004).

${ }^{6}$ M. Yoshida, S. Uemura, T. Kodzasa, T. Kamata, M. Matsuzawa, and T. Kawai, Synth. Met. 137, 967 (2003).

${ }^{7}$ X. Liu, S. H. Mohamed, J. M. Ngaruiya, M. Wuttig, and T. Michely, J. Appl. Phys. 93, 8 (2003).

${ }^{8}$ M. Shtein, J. Mapel, J. P. Benziger, and S. R. Forrest, Appl. Phys. Lett. 81, 2 (2002).

${ }^{9}$ T. W. Kelley, L. D. Boardman, T. D. Dunbar, D. V. Muyres, M. J. Pellerite, and T. P. Smith, J. Phys. Chem. B 107, 5877 (2003).

${ }^{10}$ L. L. Kosbar, C. D. Dimitrakopoulos, and D. J. Mascaro, Mater. Res. Soc. Symp. Proc. 665, C10.6.1 (2001).

${ }^{11}$ S. Verlaak, S. Steudel, D. Janssen, and P. Heremans, Phys. Rev. B 68, 195409 (2003).

${ }^{12}$ I. P. M Bouchons, W. A. Schoonveld, J. Krijmoeth, and T. M. Klapwijk, Synth. Met. 104, 175 (1999).

${ }^{13}$ S. Verlaak, Ph.D. thesis, Katholieke Universiteit Leuven, 2004. 